\title{
Design and Control of Compliantly Actuated Bipedal Running Robots: Concepts to Exploit Natural System Dynamics
}

\author{
Dominic Lakatos, Christian Rode, Andre Seyfarth, and Alin Albu-Schäffer
}

\begin{abstract}
Biped running can be conceptually reduced to a set of simple and quasi-independent tasks such as weight bearing, upper-body balancing, and energy injection through ankle push-off. We show in this paper that by appropriately designing multi-articular elastic actuators for biped robots in a manner inspired by human biomechanics, these tasks can be favorably expressed in a set of coordinates, in which the system is elastically decoupled. In these coordinates, the robot can be easily controlled by a set of simple and independent control laws. By exploiting the natural dynamics of the specially designed robot, the proposed controller requires only minimal model knowledge (mainly in terms of kinematic and static parameters) and is therefore robust to model uncertainties. It requires only state measurements and no measurement or model based computation of higher order state derivatives. Moreover, since the system is operated at a frequency dictated by the natural resonance, the running gait is energy efficient and resembles to a large extent natural human motion. Simulations validate the concept and demonstrate the independence of the approach from the knowledge of dynamics parameters.
\end{abstract}

\section{INTRODUCTION}

To cope with limitations of energy, peak force and velocity, humans exploit their passive-dynamics to perform highly dynamical walking and running tasks in an effective and highly efficient manner [1]. Since both, energy sources and peak-power of actuators, are limited in humanoid robots too, understanding and transferring this knowledge to robotic design and control is crucial. From experimentally supported hypotheses of biologists which reveal that high-dimensional, nonlinear dynamics in animals can be represented by simple template dynamics [2], it becomes evident that a robotic design capable of performing efficient locomotion needs to address two complementary aspects: the basic dynamics of an efficient gait and a mechatronic implementation that intrinsically fits to this specific dynamics. These two "ingredients" lead to what we call natural dynamics exploitation.

The approaches based on the inverted pendulum model and the zero moment point (ZMP) [3], [4], [5] are mainly intended for walking gaits. These approaches apply to fully actuated rigid robots which cannot handle high peak forces as potentially appearing in running gaits. To evolve from walking to running, the introduction of elastic actuation elements helps to reduce the impact forces and offers energy saving

D. Lakatos and A. Albu-Schäffer are with the Institute of Robotics and Mechatronics, German Aerospace Center (DLR), D-82234 Oberpfaffenhofen, Germany.

C. Rode is with the Department of Motion Science, Friedrich Schiller Universität Jena, D-07749 Jena, Germany.

A. Seyfarth is with the Lauflabor at Technische Universität Darmstadt, D-64289 Darmstadt, Germany.

Contact: dominic.lakatosedr.de capabilities for bouncing and swing motions of the legs [6]. On the basis of these insights, the conceptual spring loaded inverted pendulum (SLIP) models [7], [8], [9], [10] or the compliant hybrid zero dynamics framework [11] have been introduced. Both, the template dynamics of SLIP model and the hybrid zero dynamics resulting from a virtual constraint substantially differ from the dynamics of a humanoid robot with segmented legs having non-negligible mass. Therefore, the desired SLIP behavior has to be imposed to the plant, e.g., by feedback linearization [11] or virtual model control [12], [13], [14]. The robotic control implementations of legged locomotion hence require to substantially change the dynamics of the plant.

A main conflict in the above implementations is that the template dynamics considers elasticities, but the plant has usually none (or intrinsic elasticities are not in the required form). In our recent work [15], [16], [17], [18], [19], [20], we developed concepts to excite the intrinsic oscillatory dynamics of compliantly actuated mechanical systems [21], [22] for cyclic tasks. The control approaches apply to systems in which balancing is of minor importance such as multi-legged hopping robots and thereby the focus is on exploiting the natural dynamics of the plant.

This work aims at extending the concepts and designing a robot system capable of efficient bipedal walking and running. This requires to address several tasks such as bouncing, balancing, and swinging for foot placement [23], simultaneously. The contribution of this paper is twofold: first, we propose a task-oriented system design which allows to intrinsically access and control the relevant dynamics quantities independently. Second, we introduce a control framework, which achieves the locomotion tasks while changing the dynamics of the plant only to a minimum extent and involving neither a dynamic model nor higher derivatives of measured states in the feedback loops. Due to the reduced requirements regarding model knowledge and measured state derivatives compared to existing approaches [14], [11], this method has the potential of increased feasibility and robustness in the practical implementation.

The paper is organized as follows: Sect. II motivates the approach and introduces the basic concepts. The system design is proposed in Sect. III. Then, Sect. IV introduces the transformation for the task-oriented coordinates which forms the basis for the controller design in Sect. V. Simulations of the control system are provided in Sect. VI. A brief conclusion is given in Sect. VII. 


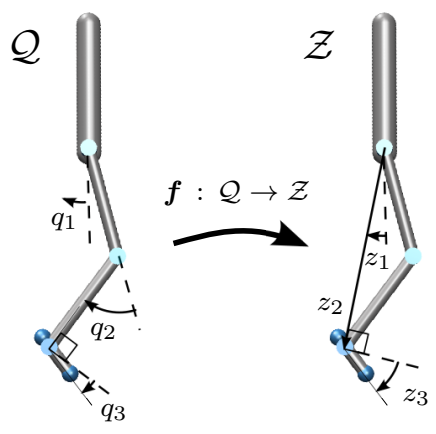

Fig. 1. Coordinate transformation

\section{CONCEPTS}

The basic idea of the approach is twofold. It combines a task-oriented system design and a "minimally invasive" control approach. A main requirement of the controller design is robustness against parameter uncertainties and measured signal noise, which can be achieved by (adaptive) methods exciting the intrinsic plant dynamics [17], but which in turn require the plant to fit to the dynamics of the task.

\section{A. Polar coordinates}

The design of the bipedal locomotion system is the result of synthesizing the following subtasks of the gait cycle:

- controlling the trunk orientation;

- foot placement;

- weight bearing via the leg axis during the stance phase;

- flexing the leg to ensure ground clearance during the swing phase;

- energy injection to sustain the limit cycle of the walking and/or running task;

- push-off to actively induce the flight phase.

Some of these subtasks such as trunk orientation and weight bearing have to be fulfilled simultaneously and independently.

From the viewpoint of versatile system design, the ground clearance requirement (on uneven terrain with obstacles) already excludes Raibert's pogo-stick design [24] and suggests a human-like segmented leg design. Further, segmented legs can adjust their stiffness by changing their configuration (bent, extended). The generalized joint coordinates describing the configuration of the segmented leg do not coincide with coordinates representing the above subtasks appropriately. Therefore, so-called task oriented coordinates are introduced (see, Fig. 1):

- $z_{1}$ represents the rotation of the leg axis w.r.t. the trunk. This coordinate is responsible to control the trunk orientation during the stance phase and to control the foot placement during the swing phase.

- $z_{2}$ represents the distance between the ankle and hip joint. With this coordinate the leg flexion is controlled.

- $z_{3}$ represents the rotation of the foot w.r.t. the leg axis. This coordinate direction can be used to inject energy during the push-off phase.

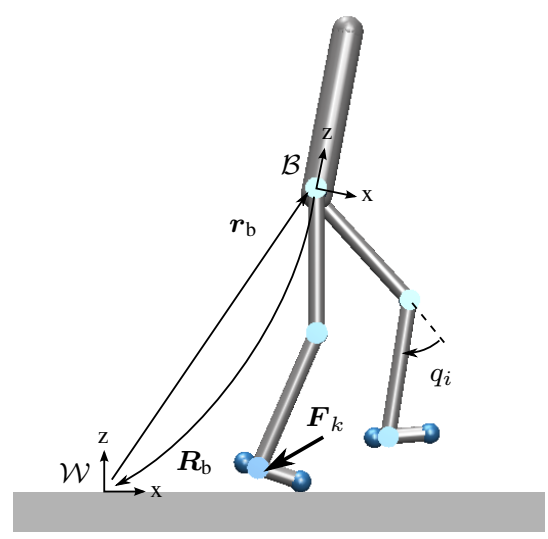

Fig. 2. Kinematic structure.

In addition to this kinematics concept, the actuator architecture is of tantamount importance. Weight bearing with reduced contact forces and efficient leg swinging during the stance and swing phase as well as reducing the impact forces of the ground contact demands compliant actuators [6]. However, the nonlinear kinematics of the segmented leg combined with linear joint elasticities lead to nonlinear coupled elastic behavior in the above mentioned taskoriented coordinates. The requirement that some of the above subtasks have to be fulfilled simultaneously and independently together with the introduction of the task-oriented intrinsically decoupled coordinates lead to a specific (compliant) actuator architecture. This functional decoupling in task coordinates is achieved by so-called biarticular muscles which result in an elastic coupling of adjacent joints. The specific actuator architecture and requirements on the rigidbody system kinematics which comply with the definition of task-oriented coordinates, result in a system design which can be controlled with simple structured, low effort, and very robust feedback control methods.

\section{SYSTEM DESIGN}

\section{A. Rigid body model}

Consider the planar bipedal floating base system with a kinematic structure as shown in Fig. 2. The position and orientation of the base link frame $\{\mathcal{B}\}$ with respect to a world coordinate system $\{\mathcal{W}\}$ (in the plane) is described by $\boldsymbol{r}_{\mathrm{b}} \in \mathbb{R}^{2}$ and $\boldsymbol{R}_{\mathrm{b}}=\boldsymbol{R}_{\mathrm{b}}(\phi) \in S O(1)$, respectively, and the configuration of the two legs is given by the joint coordinates $\boldsymbol{q} \in \mathcal{Q} \subset \mathbb{R}^{6}$. One rotational degree of freedom is considered for the hip, knee, and ankle joint of each leg (Fig. 1, left), respectively. The generalized velocity of the complete system $\boldsymbol{v}=\left(\dot{\boldsymbol{r}}_{\mathrm{b}}^{T}, \omega, \dot{\boldsymbol{q}}^{T}\right)^{T}$ is composed of the translational velocity $\dot{\boldsymbol{r}}_{\mathrm{b}}$ and the angular velocity $\omega=\dot{\phi} \in \mathbb{R}$ of the floating base, and the joint velocity $\dot{\boldsymbol{q}} \in \mathbb{R}^{6}$ for all actuated joints. In the following, we consider the dynamic system satisfying

$$
\begin{array}{r}
\boldsymbol{M}\left(\boldsymbol{q}, \boldsymbol{R}_{b}\right) \dot{\boldsymbol{v}}+\boldsymbol{p}\left(\boldsymbol{q}, \boldsymbol{v}, \boldsymbol{R}_{b}\right) \\
=\left(\begin{array}{c}
\mathbf{0} \\
\boldsymbol{\tau}-\boldsymbol{d}(\dot{\boldsymbol{q}})
\end{array}\right)+\sum_{k} \boldsymbol{J}_{k}(\boldsymbol{q})^{T} \boldsymbol{F}_{k},
\end{array}
$$




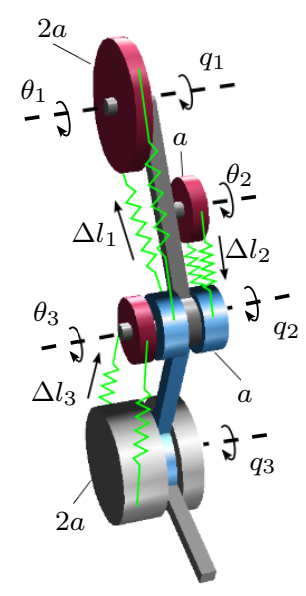

Fig. 3. Elastic actuator architecture. Red pulleys represent independent degrees of freedom represented by additional motor coordinates $\boldsymbol{\theta} \in \mathbb{R}^{3}$. $\theta_{1}$ is measured w. r. t. the trunk, $\theta_{2}$ and $\theta_{3}$ are measured w. r. t. the thigh link. The rotation of the thigh w.r.t. the trunk is measured by $q_{1}$. The two pulleys in blue are rigidly connected to the shank (blue). Thereby, the relative rotation of the shank w.r.t. the thigh is measured by $q_{2}$. The grey pulley is rigidly connected to the foot which has a rotational degree of freedom relative to the shank, measured by $q_{3}$. Each $\theta_{i}$ can be equally represented by the coordinates $\Delta l_{i}$ which represent the deflections of springs. Thereby, $\Delta l_{1}$ corresponds to spring implementing an elastic coupling of $\theta_{1}, q_{1}$ and $q_{2}$ with a $2 / 1$ coupling ratio. The coordinate $\Delta l_{2}$ corresponds to a $1 / 1$ coupling spring of $\theta_{2}$ and $q_{2}$. Finally, $\Delta l_{3}$ implements a $1 / 2$ coupling of $\theta_{3}, q_{2}$ and $q_{3}$.

where $\boldsymbol{M}\left(\boldsymbol{q}, \boldsymbol{R}_{b}\right) \in \mathbb{R}^{9 \times 9}$ represents the inertia matrix, $\boldsymbol{p}\left(\boldsymbol{q}, \boldsymbol{v}, \boldsymbol{R}_{b}\right) \in \mathbb{R}^{9}$ represents the vector of Coriolis, centrifugal and gravity forces, and $\boldsymbol{d}(\dot{\boldsymbol{q}})$ is a damping force satisfying $\boldsymbol{d}(\dot{\boldsymbol{q}})^{T} \dot{\boldsymbol{q}}>0, \forall \dot{\boldsymbol{q}} \neq \mathbf{0}$. The most right term in (1) accounts for contact wrenches $\boldsymbol{F}_{k} \in \mathbb{R}^{3}$ acting at foot $k \in\{1, \mathrm{r}\}$. Thereby, the transposed of the Jacobian matrices $\boldsymbol{J}_{k}(\boldsymbol{q})$ maps the contact wrenches to the generalized forces of the bodies.

\section{B. Elastic actuator design}

In contrast to many bipedal locomotion approaches introduced in literature so far [3], [4], [5], [14], [25], we consider compliant actuators in the joints. In particular, this section derives the compliant actuator architecture which implements the decoupling in the task-oriented coordinates introduced in Sect. II which will be analyzed in detail in Sect. IV. Introducing motor positions $\boldsymbol{\theta} \in \mathbb{R}^{3}$ as additional system states for each leg, the torques

$$
\boldsymbol{\tau}=\boldsymbol{\psi}(\boldsymbol{\theta}, \boldsymbol{q})
$$

in (1) represent generalized forces produced by springs in the joints. This model already considers classical approximations for robotic systems [26], [27] such that $\boldsymbol{\theta}$ can be considered as control input.

In the following, we derive the compliant actuator architecture which produces the elastic torques in (2) such that it complies with the concepts proposed in Sect. II. To this end, consider a single leg with configuration variables $\boldsymbol{q} \in \mathbb{R}^{3}$ and motor positions $\boldsymbol{\theta} \in \mathbb{R}^{3}$. The potential

$$
U(\boldsymbol{\theta}, \boldsymbol{q})=U_{1}\left(\Delta l_{1}\right)+U_{2}\left(\Delta l_{2}\right)+U_{3}\left(\Delta l_{3}\right)
$$

comprises functions which depend on intermediate variables

$$
\begin{aligned}
\Delta l_{1}\left(\theta_{1}, \boldsymbol{q}\right) & =\left[2\left(q_{1}-\theta_{1}\right)+q_{2}\right] a, \\
\Delta l_{2}\left(\theta_{2}, q_{2}\right) & =\left(q_{2}-\theta_{2}\right) a, \\
\Delta l_{3}\left(\theta_{3}, \boldsymbol{q}\right) & =\left[\left(q_{2}-\theta_{3}\right)+2 q_{3}\right] a,
\end{aligned}
$$

with the lever arm constant $a>0$, representing generalized deflections of elastic elements (Fig. 3). The functions $U_{1}, U_{2}, U_{3}$ in (3) are assumed to be positive definite, i.e., $\forall \Delta l_{i} \neq 0, U_{i}\left(\Delta l_{i}\right)>0, i=1,2,3$. Then, as a consequence of (4)-(6), the potential (3) is positive definite in a sense that

$$
\exists \boldsymbol{\theta}_{0}, \boldsymbol{q}_{0} \in \mathbb{R}^{3}, \forall \boldsymbol{\theta} \neq \boldsymbol{\theta}_{0}, \boldsymbol{q} \neq \boldsymbol{q}_{0}, U(\boldsymbol{\theta}, \boldsymbol{q})>0 .
$$

In the following and without loss of generality, we consider quadratic functions

$$
\begin{aligned}
U_{1}\left(\Delta l_{1}\right) & =\frac{1}{2} k_{1}\left(\Delta l_{1}\right)^{2}, \\
U_{2}\left(\Delta l_{2}\right) & =\frac{1}{2} k_{2}\left(\Delta l_{2}\right)^{2}, \\
U_{2}\left(\Delta l_{3}\right) & =\frac{1}{2} k_{3}\left(\Delta l_{3}\right)^{2},
\end{aligned}
$$

in (3), where $k_{1}, k_{2}, k_{3}>0$ are spring constants. The generalized spring force (for a single leg) can be derived from the resulting potential, i.e.,

$$
\begin{aligned}
\boldsymbol{\tau} & =-\frac{\partial U(\boldsymbol{\theta}, \boldsymbol{q})}{\partial \boldsymbol{q}} \\
& =\left(\begin{array}{c}
2 k_{1}\left[2\left(\theta_{1}-q_{1}\right)-q_{2}\right] \\
k_{1}\left[2\left(\theta_{1}-q_{1}\right)-q_{2}\right]+k_{2}\left(\theta_{2}-q_{2}\right)+k_{3}\left[\theta_{3}-q_{2}+2 q_{3}\right] \\
2 k_{3}\left[\theta_{3}-q_{2}+2 q_{3}\right]
\end{array}\right) a^{2} .
\end{aligned}
$$

The Hessian of the potential, also referred to as the mechanical stiffness, takes the form

$$
\begin{aligned}
\boldsymbol{K} & =\frac{\partial^{2} U(\boldsymbol{\theta}, \boldsymbol{q})}{\partial \boldsymbol{q}^{2}} \\
& =\left[\begin{array}{ccc}
4 k_{1} & 2 k_{1} & 0 \\
2 k_{1} & k_{1}+k_{2}+k_{3} & 2 k_{3} \\
0 & 2 k_{3} & 4 k_{3}
\end{array}\right] a^{2} .
\end{aligned}
$$

The structure of the stiffness matrix indicates an elastic coupling of the joint coordinates $q_{1}, q_{2}$ and $q_{2}, q_{3}$, respectively. Note that this is not a result of the particular choice of the quadratic functions (7)-(9). The elastic coupling results for any potential (3) defined by generalized deflections introduced in (4)-(6).

\section{COORDINATE TRANSFORMATION}

This section derives the transformation to the task-oriented coordinates which together with the elastic actuator architecture proposed in Sect. III-B form the basis to effectively control bipedal gaits.

Consider again a single leg, segmented in thigh, shank, and foot (Fig. 1, left). For reasons discussed later, assume that the segment lengths of thigh and shank are equal and denoted by $b>0$. The goal of the coordinate transformation is to describe the configuration of the leg by coordinates representing the length of the leg $z_{2}$ (distance between tip of the shank and hip), the orientation of this axial leg w.r.t. the 
trunk $z_{1}$, and the orientation of the foot w.r.t. the axial leg $z_{3}$ (Fig. 1, right). To this end, consider as an intermediate computation step the position of the tip of the shank w.r.t. a coordinate frame attached to the trunk and located at the hip:

$$
\begin{aligned}
& x^{\mathrm{f}}(\boldsymbol{q})=-\left[\sin \left(q_{1}+q_{2}\right)+\sin \left(q_{1}\right)\right] b, \\
& z^{\mathrm{f}}(\boldsymbol{q})=-\left[\cos \left(q_{1}+q_{2}\right)+\cos \left(q_{1}\right)\right] b .
\end{aligned}
$$

Then, the new coordinates result by transforming from Cartesian to polar coordinates, i. e.

$$
\boldsymbol{z}=\boldsymbol{f}(\boldsymbol{q})=\left(\begin{array}{c}
\arctan \left(\frac{\sin \left(q_{1}+q_{2}\right)+\sin \left(q_{1}\right)}{\cos \left(q_{1}+q_{2}\right)+\cos \left(q_{1}\right)}\right) \\
b \sqrt{2+2 \cos \left(q_{2}\right)} \\
\sum_{i=1}^{3} q_{i}-\arctan \left(\frac{\sin \left(q_{1}+q_{2}\right)+\sin \left(q_{1}\right)}{\cos \left(q_{1}+q_{2}\right)+\cos \left(q_{1}\right)}\right)
\end{array}\right)
$$

where $z \in \mathcal{Z} \subset \mathbb{R}^{3}$, with corresponding Jacobian matrix

$$
\boldsymbol{J}(\boldsymbol{q})=\frac{\partial \boldsymbol{f}(\boldsymbol{q})}{\partial \boldsymbol{q}}=\left[\begin{array}{ccc}
1 & \frac{1}{2} & 0 \\
0 & -\frac{b \sin \left(q_{2}\right)}{\sqrt{2+2 \cos \left(q_{2}\right)}} & 0 \\
0 & \frac{1}{2} & 1
\end{array}\right] .
$$

\section{A. Properties of the coordinate transformation}

In the following we will summarize some important properties of the coordinate transformation (14):

(P1) From (15) it can be seen that the mapping (14) is invertible everywhere except at $q_{2}=0, \pi, 2 \pi, \ldots$.

(P2) The mapping $z_{2}=f_{2}\left(q_{2}\right)$ depends only on $q_{2}$ not on $q_{1}, q_{3}$. This will be important for the definition of the control inputs considered next.

(P3) In the new coordinate system defined by (14), the generalized spring force resulting from (3)-(6) is decoupled in a sense that the matrix

$$
\boldsymbol{K}_{z}=\left(\frac{\partial \boldsymbol{f}(\boldsymbol{q})}{\partial \boldsymbol{q}}\right)^{-T} \frac{\partial^{2} U(\boldsymbol{\theta}, \boldsymbol{q})}{\partial \boldsymbol{q}^{2}}\left(\frac{\partial \boldsymbol{f}(\boldsymbol{q})}{\partial \boldsymbol{q}}\right)^{-1}
$$

is diagonal.

In case of the quadratic functions (7)-(8), property (P3) can be verified by direct calculation, i.e.,

$$
\boldsymbol{K}_{z}=\left[\begin{array}{ccc}
k_{1} & 0 & 0 \\
0 & \frac{k_{2}}{b^{2}\left[2-2 \cos \left(q_{2}\right)\right]} & 0 \\
0 & 0 & k_{3}
\end{array}\right] 4 a^{2} .
$$

The above properties will be exploited especially in the definitions of the control inputs.

\section{B. Control inputs}

The controller introduced in the next section will be formulated w. r. t. the functionally decoupled coordinate system defined by (14). Thereby, a requirement of the specific system design is that combinations of torques and equilibrium positions of the springs can be considered as independent control inputs. For instance, it could be required to control the trunk orientation with a PD controller via the hip torque $\tau_{z, 1}$ while independently bearing the weight via the equilibrium position of the axial leg $\bar{z}_{2}$. In the following, the control input combinations are derived which are required to implement the subtasks explained in Sect. II.

The physical control inputs of the plant are the motor positions $\boldsymbol{\theta}$. To define the equilibrium position $\overline{\boldsymbol{z}} \in \mathcal{Z} \subset \mathbb{R}^{3}$ as control input, we are seeking motor positions $\boldsymbol{\theta}=\boldsymbol{\theta}(\overline{\boldsymbol{z}})$ corresponding to $\bar{z}$. This can be reached in two steps. First, consider the inverse of (14)

$$
\boldsymbol{q}=\boldsymbol{g}(\boldsymbol{z})=\boldsymbol{f}(\boldsymbol{z})^{-1}
$$

where $\boldsymbol{q} \in \mathcal{Q} \subset \mathbb{R}^{3}$, such that we can compute an equivalent equilibrium position w.r.t. the $\mathcal{Q}$ coordinate system

$$
\overline{\boldsymbol{q}}=\boldsymbol{g}(\overline{\boldsymbol{z}}) .
$$

Then in a second step, equivalent motor positions $\boldsymbol{\theta}=\boldsymbol{\theta}(\overline{\boldsymbol{q}})$ can be computed using the equilibrium condition

$$
\frac{\partial U(\boldsymbol{\theta}, \overline{\boldsymbol{q}})}{\partial \overline{\boldsymbol{q}}}=\mathbf{0}
$$

or equivalently due to the positive definiteness of the functions $U_{i}\left(\Delta l_{i}\right)$ and the definition of the generalized deflections (4)-(6),

$$
\begin{aligned}
\theta_{1}(\overline{\boldsymbol{q}}) & =\bar{q}_{1}+\frac{\bar{q}_{2}}{2}, \\
\theta_{2}\left(\bar{q}_{2}\right) & =\bar{q}_{2}, \\
\theta_{3}(\overline{\boldsymbol{q}}) & =\bar{q}_{2}+2 \bar{q}_{3} .
\end{aligned}
$$

To define the torque of the hip and the equilibrium positions of the leg axis and the foot $\left(\tau_{z, 1}, \bar{z}_{2}, \bar{z}_{3}\right)$ as control input, we are seeking motor positions $\boldsymbol{\theta}=\boldsymbol{\theta}\left(\tau_{z, 1}, \bar{z}_{2}, \bar{z}_{3}\right)$. From the transformation law of generalized forces from the $\mathcal{Z}$ to the $\mathcal{Q}$ coordinate system

$$
\boldsymbol{\tau}=\boldsymbol{J}(\boldsymbol{q})^{T} \boldsymbol{\tau}_{z}
$$

and the structure of the Jacobian matrix (15) it can be seen that $\tau_{1}=\tau_{z, 1}$. Then, given $\boldsymbol{q}$ and since the only motor position which appears in $\Delta l_{1}\left(\theta_{1}, \boldsymbol{q}\right)$ (defined in (4)) is $\theta_{1}$, the equation

$$
-\frac{\partial U(\boldsymbol{\theta}, \boldsymbol{q})}{\partial q_{1}}\left(\theta_{1}, \boldsymbol{q}\right)=\tau_{1}\left(\theta_{1}\right)
$$

can be uniquely solved for $\theta_{1}=\theta_{1}\left(\tau_{z, 1}\right)$, since $\tau_{1}\left(\theta_{1}\right)$ is strictly monotonic in $\theta_{1}$. To relate the equilibrium positions $\bar{z}_{2}, \bar{z}_{3}$ to the remaining motor positions $\theta_{2}, \theta_{3}$, the equilibrium position $\bar{z}_{1}$, corresponding to $\left(\tau_{z, 1}, \bar{z}_{2}, \bar{z}_{3}\right)$ has to be computed. This can be done by considering the second row of (16), (19), and the first row of (14), i.e.,

$$
\begin{aligned}
& \bar{q}_{2}=g_{2}\left(\bar{z}_{2}\right), \\
& \bar{q}_{1}=-\frac{\bar{q}_{2}-2 \theta_{1}}{2}, \\
& \bar{z}_{1}=f_{1}\left(\bar{q}_{1}, \bar{q}_{2}\right) .
\end{aligned}
$$

Once $\bar{z}_{1}$ is obtained, the remaining motor positions $\theta_{2}, \theta_{3}$ result by applying successively (16) and (19)-(21). As a result, $\left(\tau_{z, 1}, \bar{z}_{2}, \bar{z}_{3}\right)$ can be considered as control input.

Remark 1: The above derivation is only an example of how the control input in the $\mathcal{Z}$ coordinate system can be 
chosen independently. It can be verified that the combinations of generalized forces and equilibrium positions $\left(\bar{z}_{1}, \bar{z}_{2}, \tau_{z, 3}\right)$ and $\left(\tau_{z, 1}, \bar{z}_{2}, \tau_{z, 3}\right)$, can be considered as tantamount control inputs. Note that this is a result of the system design introduced in Sect. 3 and the particular kinematics as depicted in Fig. 1, where the segment lengths of thigh and shank are equal.

\section{CONTROLler DESIGN}

The locomotion control concept is based on biomechanics insights, special kinematics design, and our recently proposed limit cycle control method as introduced in [15], [17], [18], [20]. The controller explained next is an overall result of the system design, the task-oriented coordinate transformation, and our recently introduced model-free control concepts.

The controller is hybrid in a sense that it combines a switching logic (state machine) and continuous control laws resulting in combined finite and continuous closed-loop dynamics.

\section{A. Control actions}

Two types of control actions are considered:

1) changing the equilibrium positions of the springs;

2) implementing a PD control with gravity compensation [28, Sect. 2.2.3]:

$$
\tau_{z, 1}=\tau_{\mathrm{g}}(\phi)-k_{\mathrm{P}}\left[\phi-\phi_{\mathrm{d}}\right]-k_{\mathrm{D}} \dot{\phi}
$$

where $k_{\mathrm{P}}, k_{\mathrm{D}}>0$ are controller gains and $\tau_{\mathrm{g}}$ is the torque acting due to the weight of the trunk on the hip, or the "zero torque" control

$$
\tau_{z, 1}=\tau_{\mathrm{g}}(\phi) .
$$

The control action 1) has multiple responsibilities:

- foot placement during swing and flight phase via the control input $\bar{z}_{1}$;

- energy input by loading the spring (responsible for leg extension) via the control input $\bar{z}_{2}$ and/or energy input by push-off during the stance phase via the ankle joint considering the control input $\bar{z}_{3}$;

- controlling the ground clearance during swing phase by shortening the leg via the control input $\bar{z}_{2}$;

- fine tuning and repositioning of the foot for touch down via the control input $\bar{z}_{3}$.

Except for the latter, all the control actions of type 1) are instantaneous, i.e. the equilibrium positions $\bar{z}$ are changed stepwise.

The control action 2) is responsible for controlling the orientation of the trunk w.r.t. the ground (27) via the control input $\tau_{z, 1}$. The PD control (27) is active as long as the ground reaction forces are sufficiently hight such that friction forces support the torques in the hip, otherwise the "zero torque" mode (28) is active.

Remark 2: Note that the torque control input $\tau_{z, 1}$ is always considered in combination with the equilibrium position input $\bar{z}_{2}, \bar{z}_{3}$. This combination is only possible due to the specific elastic model (Sect. III-B) and the coordinate transformation introduced in Sect. IV.

Further, note that to any of the task-oriented coordinates one can relate a main functionality which can be controlled independently. The coordinate $z_{1}$ is responsible to control the trunk independent of the length of the leg, and the leg orientation in the swing phase. The axial leg function represented by $z_{2}$ is used for weight bearing during stance, and for ground clearance during swing. The ankle rotation represented by $z_{3}$ (possibly in combination with the axial leg function) is responsible for energy injection.

\section{B. Switching logic (state machine)}

This section derives the finite dynamics of the control system which determines the switching between different controllers and desired states. It can be interpreted as a state machine that changes finite states triggered by events. These events are only functions of the continuous system states and therefore this concept further supports the idea of exploiting the natural dynamics of the plant [17]. In the following, we present the control framework exemplary for a specific running controller. Many different controllers and parameter configurations resulting in different gaits can be considered on the basis of this framework.

For each leg we consider the following events:

- touchdown of the foot denoted by $T D$;

- threshold functions for the axial leg force $\tau_{z, 2}>$ $\epsilon_{\tau_{z} \uparrow}^{1}, \tau_{z, 2}>\epsilon_{\tau_{z} \uparrow}^{2}$, and $\tau_{z, 2} \leq \epsilon_{\tau_{z \downarrow} \downarrow}^{3}$, where the arrow $(\cdot)_{\uparrow} /(\cdot)_{\downarrow}$ indicates whether the force over-/undershoots the threshold implication;

- takeoff of the foot denoted by TO.

On the basis of the above events the state machine illustrated in Fig. 4 switches the (continuous) control actions described in Sect. V-A. Thereby, the superscripts $(\cdot)^{1}$ and $(\cdot)^{\mathrm{r}}$ indicate quantities of the left and right leg, respectively. In the following, the functionality of each finite state in Fig. 4 is described:

State 1: Right after the TD, the hip torque of the stance leg compensates only for the gravitational torque of the upper body, while the equilibrium position of the leg axis keeps the neutral position $\hat{z}_{2}^{0}=$ const.. For the swing leg, the equilibrium position of the hip rotation keeps the value of the hip position at the TO event $z_{1}(T O)$ and the equilibrium position of the leg axis keeps the parameter value $\hat{z}_{2}^{-}<\hat{z}_{2}^{0}$. The equilibrium positions of both ankle joints are such that the feet keep parallel to the ground. In particular, the equilibrium position of the stance leg ankle joint keeps the value at the entry time instance of this finite state, i.e., $-\left(\phi+z_{1}^{1}\right)^{\text {en }}$. Therefore, this state mainly assures the ground contact for the stance phase.

State 2: The assured ground contact of the stance leg is detected using the threshold event $\tau_{z, 2}>\epsilon_{\tau_{z} \uparrow}^{1}$. In this case, the upper body inclination is controlled via the hip torque of the stance leg. Additionally, the foot of the swing leg is placed by changing the equilibrium position of the spring in the hip. Note that the foot placement controls 


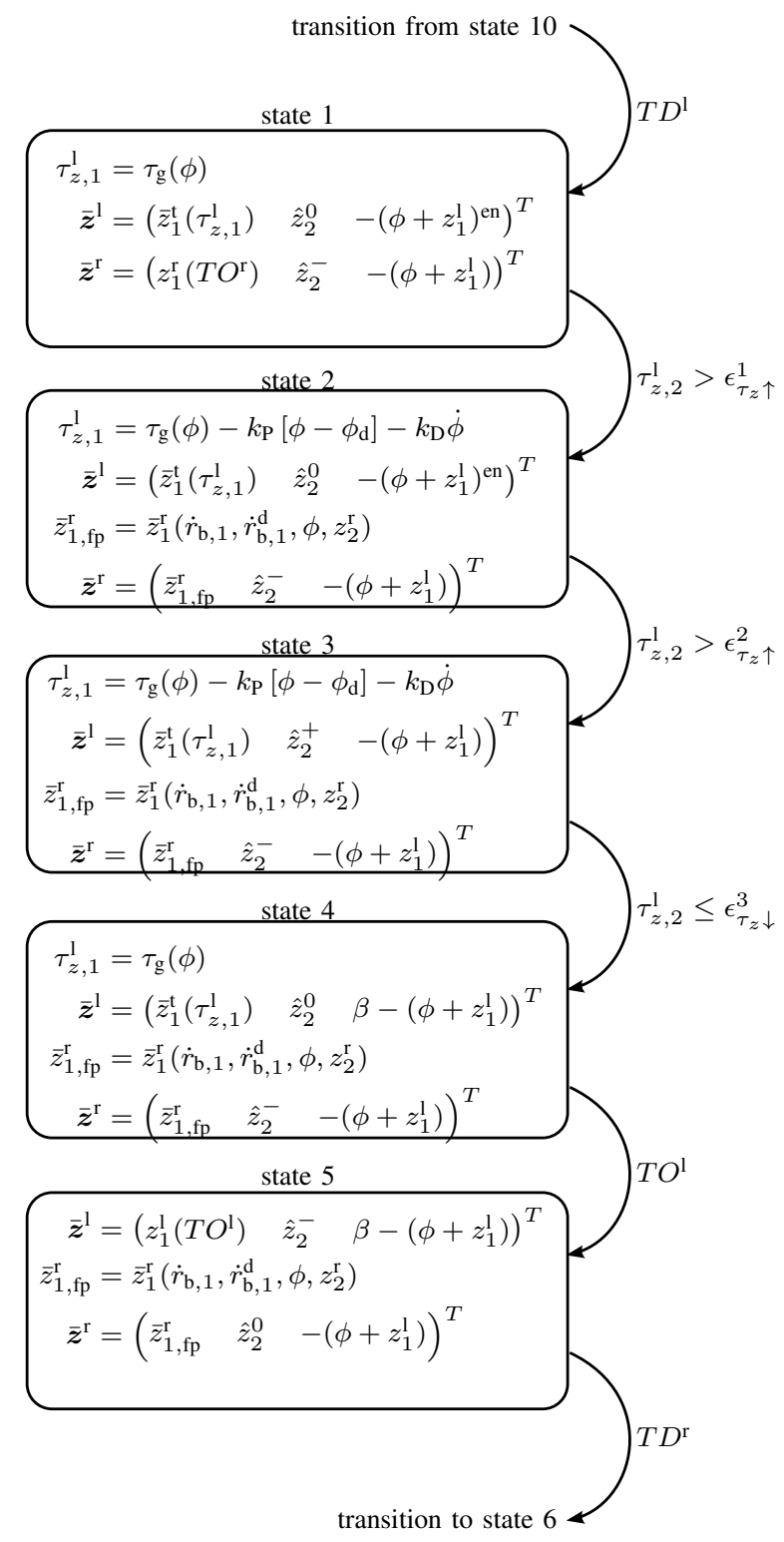

Fig. 4. State machine controlling the gate cycle. Control actions for the inputs described in Sect. IV-B are defined. Half a gate cycle is depicted, i. e.the stance and flight phase of the left leg and the swing phase of the right leg. The states 6 to 10 can be obtained by interchanging the superscripts $(\cdot)^{\mathrm{l}}$ and $(\cdot)^{\mathrm{r}}$ in the equations of states 1 to 5 . Note that the superscript $(\cdot)^{\text {en }}$ denotes the time instance of the finite state entry and the subscript $(\cdot)_{\mathrm{fp}}$ means foot placement.

the transportation velocity of the gait. The corresponding algorithm is described in Sect. V-C.

State 3: The event $\tau_{z, 2}>\epsilon_{\tau_{z} \uparrow}^{2}$, where $\epsilon_{\tau_{z} \uparrow}^{2}>\epsilon_{\tau_{z} \uparrow}^{1}$, may be used to trigger the energy injection of a superimposed (periodic) hopping motion as the limit cycle control proposed in [20]. This can be achieved by changing the equilibrium position of the leg axis to the parameter value $\hat{z}_{2}^{+}>\hat{z}_{2}^{0}$ and leads in turn to the TO of the stance leg. In case of human like walking and running, the energy injection is implemented via the ankle joint. Thereby, this finite state may be used to prepare the push-off phase.
State 4: This finite state directly induces the TO and already prepares for the ground clearance in the next swing phase by either decreasing the equilibrium position of the leg axis (of the stance leg) to $\hat{z}_{2}^{0}$ or by loading the spring corresponding to the ankle joint with a constant pretension angle $\beta>0$. Additionally, the hip torque of the stance leg compensates only for the gravitational torque of the upper body, since the ground reaction forces are approaching zero and therefore the orientation of the trunk cannot be regulated any more.

State 5: During the flight phase, the equilibrium position of the hip of the last stance leg is kept at the position $z_{1}(T O)$ at the TO event. The next swing leg is shortened by decreasing the corresponding equilibrium position to $\hat{z}_{2}^{-}$. Further, the equilibrium position of the landing leg is changed to the neutral position $\hat{z}_{2}^{0}=$ const..

States 6 to 10: The finite states 6 to 10 can be derived analogously to states 1 to 5 by interchanging the superscripts $(\cdot)^{1}$ and $(\cdot)^{\mathrm{r}}$.

\section{Landing foot placement}

The control system described above consists of two main energy sources. One is step-wise changing the equilibrium of the ankle joint. This source introduces elastic energy into the system. The other energy source is the placement of the landing foot. Further, energy losses are due to friction and damping. It is evident that to achieve steady-state locomotion such as running at constant forward velocity, the energy input and the dissipation in the gait cycle have to be balanced. As shown in [24], the foot placement can be considered as input to control the forward velocity of the gait and therefore to control the net energy exchange. The following foot placement algorithm is based on [24]:

$$
\bar{z}_{1}\left(\dot{r}_{\mathrm{b}, 1}, \dot{r}_{\mathrm{b}, 1}^{\mathrm{d}}, \phi, z_{2}\right)=-\phi-\sin ^{-1}\left(\frac{x_{\mathrm{f}}\left(\dot{r}_{\mathrm{b}, 1}, \dot{r}_{\mathrm{b}, 1}^{\mathrm{d}}\right)}{z_{2}}\right) \text {, }
$$

where

$$
x_{\mathrm{f}}\left(\dot{r}_{\mathrm{b}, 1}, \dot{r}_{\mathrm{b}, 1}^{\mathrm{d}}\right)=\frac{\dot{r}_{\mathrm{b}, 1}^{\text {mean }} T_{\mathrm{s}}}{2}+k_{\mathrm{v}}\left(\dot{r}_{\mathrm{b}, 1}^{\text {mean }}-\dot{r}_{\mathrm{b}, 1}^{\mathrm{d}}\right) .
$$

Herein, $T_{\mathrm{s}}=t(T O)-t(T D)$ represents the duration of the stance phase and

$$
\dot{r}_{\mathrm{b}, 1}^{\text {mean }}=\frac{1}{T_{\mathrm{s}}} \int_{t(T D)}^{t(T O)} \dot{r}_{\mathrm{b}, 1}(t) \mathrm{d} t .
$$

The second term in (30) is a regulation term where $\dot{r}_{\mathrm{b}, 1}^{\mathrm{d}}$ represents the desired forward velocity of the upper body and $k_{\mathrm{v}}$ is a positive gain.

Note that $T_{s}$ and $\dot{r}_{\mathrm{b}, 1}^{\text {mean }}$ depend on the solution of the differential equation representing the stance dynamics, which is not a priori known. Starting with an initial guess, the values of the previous stance phase can be used to place the foot for the next landing leg.

\section{Simulations}

This section exemplifies the working principle of the control system and validates the effectiveness of the approach in simulation. 


\section{A. Plant parameters}

We consider a human-like mass distribution with a total body mass $m=80 \mathrm{~kg}$, comprising $0.75 \mathrm{~m}$ for the trunk, $0.0625 \mathrm{~m}$ for each thigh, $0.0375 \mathrm{~m}$ for each shank, and $0.025 \mathrm{~m}$ for each foot. Thighs and shanks have segment lengths of $0.5 \mathrm{~m}$. Each foot has a segment length of $0.2 \mathrm{~m}$. The ankle joint of each foot is located at $25 \%$ of the foot length measured w.r.t. the heel. The center of mass (CoM) of the trunk is $0.3 \mathrm{~m}$ above the hip. The CoM of thighs and shanks are at $40 \%$ of the segments lengths (proximal) and the CoM of each foot is at $30 \%$ of the forefoot length measured w. r. t. the ankle joint. We consider a dimensionless lever arm constant $a=1$ such that $k_{1}=200 \mathrm{Nm} / \mathrm{rad}$, $k_{1}=1000 \mathrm{Nm} / \mathrm{rad}$, and $k_{1}=600 \mathrm{Nm} / \mathrm{rad}$ have the physical unit of rotational stiffness. For the link side damping the same structure as for the stiffness matrix (11) with $d_{1}=d_{3}=10 \mathrm{Nms} / \mathrm{rad}$, and $d_{2}=25 \mathrm{Nms} / \mathrm{rad}$ is assumed.

\section{B. Controller parameters}

The parameters of the trunk controller have been chosen $k_{\mathrm{P}}=1000 \mathrm{Nm} / \mathrm{rad}$ and $k_{\mathrm{D}}=200 \mathrm{Nms} / \mathrm{rad}$ with a reference value $\phi^{\mathrm{d}}=0$. For the leg axis, the threshold values $\epsilon_{\tau_{z} \uparrow}^{1}=$ $400 \mathrm{~N}, \epsilon_{\tau_{z} \uparrow}^{1}=1150 \mathrm{~N}$, and $\epsilon_{\tau_{z} \downarrow}^{1}=600 \mathrm{~N}$, and the amplitude values $\hat{z}_{2}^{-}=0.9 \cdot 2 b$ and $\hat{z}_{2}^{0}=\hat{z}_{2}^{+}=0.99 \cdot 2 b$, where $b=$ $0.5 \mathrm{~m}$ have been considered. The push-off amplitude for the ankle joint has been chosen $\beta=1 / 6 \pi$. The proportional gain of the velocity controller (30) has been chosen $k_{\mathrm{v}}=0.2 \mathrm{~s}$. All controller parameters have been chosen manually.

\section{Simulation setup}

The control system dynamics have been simulated using the articulated body algorithm [29] and a compliant ground contact model [30] with a Coulomb friction coefficient $\mu=$ 0.75. All simulations started with an initial guess of the stance duration $T_{\mathrm{s}}=0.2 \mathrm{~s}$. On the basis of this initial guess and the reference values $\phi^{d} e s$ and $\dot{r}_{\mathrm{b}, 1}^{\mathrm{d}}$, the initial condition $z_{1}^{1}(0)$ was computed applying (30). Further, $z_{2}^{1}(0)=\hat{z}_{2}^{0}$, $z_{3}^{1}(0)=-\left(\phi^{d}+z_{1}^{1}(0)\right), z_{1}^{\mathrm{r}}(0)=-z_{1}^{1}(0), z_{2}^{1}(0)=\hat{z}_{2}^{-}$, and $z_{3}^{\mathrm{r}}(0)=-\left(\phi^{d}+z_{1}^{\mathrm{r}}(0)\right)$. These initial conditions were transformed to the $\mathcal{Q}$ coordinate system using (16). Then, the initial motor positions result from (19)-(21). The initial conditions of the trunk are chosen such that for $\phi(0)=$ $\phi^{\mathrm{d}}$ the landing foot almost touches the ground. All initial conditions at velocity level except the vertical velocity of the trunk $\left(\dot{r}_{\mathrm{b}, 1}=\dot{r}_{\mathrm{b}, 1}^{\mathrm{d}}\right)$ were zero. The resulting initial value problem has been integrated using a variable step solver of MATLAB/Simulink with a maximum step size of $0.01 \mathrm{~s}$.

\section{Results}

The simulation demonstrates the periodic steady-state behaviour of the closed-loop system. The desired forward velocity was set to $\dot{r}_{\mathrm{b}, 1}^{\mathrm{d}}=2 \mathrm{~m} / \mathrm{s}$. Fig. 5 shows a stroboscopic picture of a complete, simulated gait cycle. Fig. 6 shows the path of the configuration variables of one leg for five cycles in the steady-state phase of the running motion. From the latter plot the periodicity of the motion can be clearly seen.

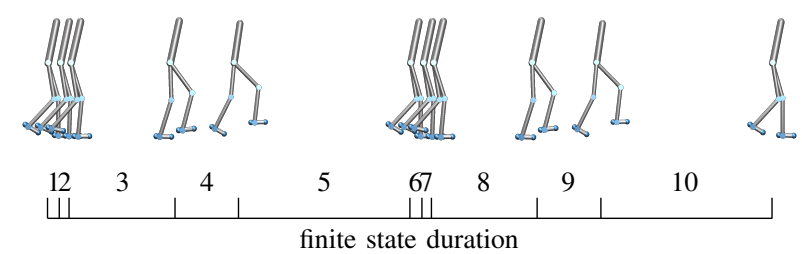

Fig. 5. A complete gait cycle in the steady-state phase of the running simulation. The motion is depicted over time. Snapshots of the bipedal system are shown at each transition of the finite states.

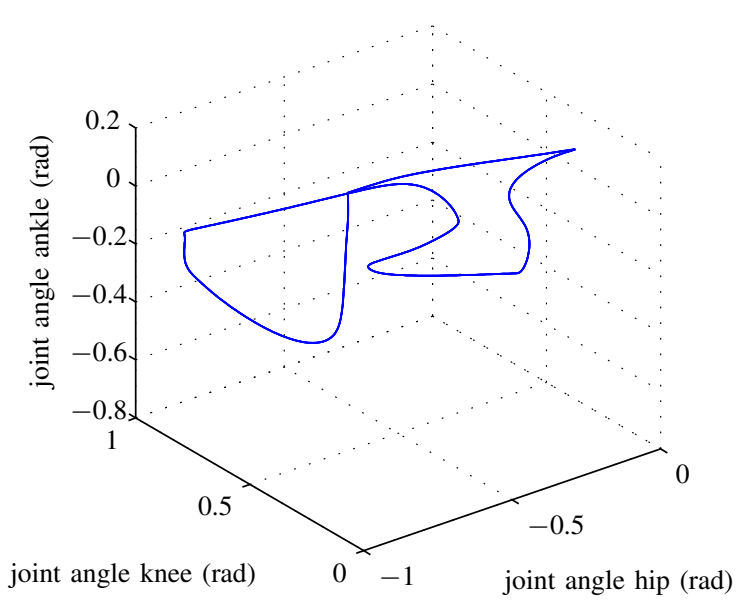

Fig. 6. Path of the configuration variables of one leg. Five cycles in the steady-state phase of the running motion are depicted.

Fig. 7 depicts the results of a convergence experiment. Two simulation runs both starting with the same initial conditions are shown. In the first simulation, the desired forward velocity is linearly increased in the time interval of $20 \mathrm{~s}$ to $30 \mathrm{~s}$. In the second simulation, the system runs uphill a slope of $8.75 \%$ while the desired forward velocity is constant. Both graphs clearly demonstrate the ability of the system to converge to different (desired) cyclic steady-states.

\section{CONCLUSION}

In our conceptual approach, biarticular series elastic actuators coupling the actuation of adjacent joints are exploited for the generation of a stable running pattern. Our selected leg segmentation (thigh and shank of equal length) and lever arm ratios of the biarticular actuators (2/1 hip to knee and ankle to knee, respectively) effectively decouple the tasks of balancing the trunk and orienting the leg in the swing phase from load carrying. Proposed control variables are associated with these distinct functions of the leg. Neutral motor positions of compliant actuators are independent of each other, hence simplifying control. This mapping of control variables and leg functions yields a number of benefits. For example, since the mapping stems from the morphology of the leg, the concept does not constrain the choice of actuators. Also, our approach enables the application of simple control principles developed for telescopic legs [24] to beneficial segmented legs. Simulations demonstrate that stable running on level ground and uphill $(8.75 \%)$ can be achieved with 


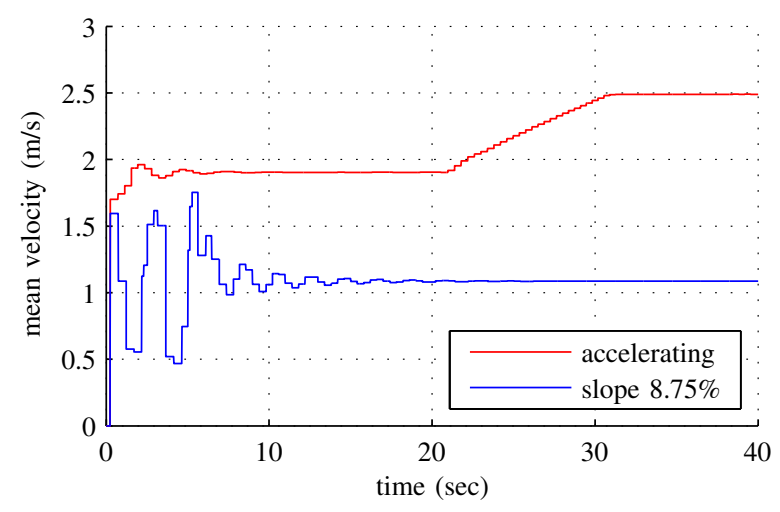

Fig. 7. Convergence behaviour of the forward velocity. The mean values of the stance phases are plotted over time. Two experiments are shown acceleration from about 2 to $2.5 \mathrm{~m} / \mathrm{s}$ and running uphill on a constant slope of $8.75 \%$.

segmented legs without changing any control parameters. This shows that the system can approach different limit cycles. Starting from this demonstration of the concept, we hope to understand the function and morphology of the human leg with possibly coupled functions better. Joint angle paths Fig. 6 can be compared with joint angle paths of humans to tune control parameters. The question how the human leg achieves linear behavior in the leg axis might in part be answered by a nonlinear knee spring compensating for the strong geometric nonlinearity in extended human leg. Concordant with a passive knee function in human running and positive work not only at the ankle but also at the hip, the concept suggests introducing energy via biarticular structures leaning the trunk forward to pump in energy, or leaning the trunk backwards to decelerate. Again, the axial function can remain in a passive bouncing mode, and biarticular structures can manage energy without hampering the axial leg function.

\section{ACKNOWLEDGMENT}

This work has been partially funded by the European Commission's Seventh Framework Programme as part of the project SAPHARI (Grant no. 287513). Additionally, this work has been partially founded by the European Commission's (Information and Communication Technologies, ICT2011.2.1) Seventh Framework Programme as part of the project BALANCE (Grand no. 601003).

\section{REFERENCES}

[1] S. Collins, A. Ruina, R. Tedrake, and M. Wisse, "Efficient bipedal robots based on passive-dynamic walkers," Science, vol. 307, no. 5712, pp. 1082-1085, 2005.

[2] R. Full and D. Koditschek, "Templates and anchors: neuromechanical hypotheses of legged locomotion on land," J. Exp. Biol., vol. 202, no. 15 , pp. 3325-3332, 1999.

[3] M. Vukobratovic and B. Borovac, "Zero-moment point - thirty five years of its life," International Journal of Humanoid Robotics, vol. 2, no. 2, pp. 225-227, 2005.

[4] S. Kajita and K. Tani, "Study of dynamic biped locomotion on rugged terrain-derivation and application of the linear inverted pendulum mode," in Proc. of IEEE Int. Conf. on Robotics and Automation, 1991.

[5] P.-B. Wieber, "Trajectory free linear model predictive control for stable walking in the presence of strong perturbations," in Proc. of IEEE/RAS Int. Conf. on Humanoid Robots, 2006.
[6] R. M. Alexander, "Three uses for springs in legged locomotion," International Journal of Robotics Research, vol. 9, no. 2, pp. 53-61, 1990.

[7] R. Blickhan, "The spring-mass model for running and hopping," Journal of Biomechanics, vol. 22, pp. 1217-1227, 1989.

[8] A. Seyfarth, H. Geyer, M. Gnther, and R. Blickhan, "A movement criterion for running." Journal of Biomechanics, vol. 35, no. 5, pp. 649-55, 2002.

[9] H. Geyer, A. Seyfarth, and R. Blickhan, "Compliant leg behavior explains basic dynamics of walking and running," Proceedings of the Royal Society B, vol. 273, pp. 2861-2867, Nov. 2006.

[10] J. Rummel, Y. Blum, H. M. Maus, C. Rode, and A. Seyfarth, "Stable and robust walking with compliant legs," in Proc. of IEEE Int. Conf. on Robotics and Automation, 2010, pp. 5250-5255.

[11] K. Sreenath, H.-W. Park, and J. W. Grizzle, "Embedding active force control within the compliant hybrid zero dynamics to achieve stable, fast running on MABEL," The International Journal of Robotics Research, 2013

[12] J. Pratt, P. Dilworth, and G. Pratt, "Virtual model control of a bipedal walking robot," in IEEE Int. Conf. on Robotics and Automation, 1997, pp. 193-198.

[13] J. Pratt and G. Pratt, "Intuitive control of a planar bipedal walking robot," in IEEE Int. Conf. on Robotics and Automation, 1998, pp. 2014-2021.

[14] M. Hutter, D. Remy, M. A. Höpflinger, and R. Siegwart, "Slip running with an articulated robotic leg," in Proc. of IEEE/RSJ Int. Conf. on Intelligent Robots and Systems, 2010, pp. 4934-4939.

[15] D. Lakatos, F. Petit, and A. Albu-Schäffer, "Nonlinear oscillations for cyclic movements in variable impedance actuated robotic arms," in IEEE Int. Conf. on Robotics and Automation, 2013.

[16] D. Lakatos, G. Garofalo, F. Petit, C. Ott, and A. Albu-Schäffer, "Modal limit cycle control for variable stiffness actuated robots," in IEEE Int. Conf. on Robotics and Automation, 2013.

[17] D. Lakatos, M. Görner, F. Petit, A. Dietrich, and A. Albu-Schäffer, "A modally adaptive control for multi-contact cyclic motions in compliantly actuated robotic systems," in Proc. IEEE/RSJ Int. Conf. on Intelligent Robots and Systems, 2013, pp. 5388-5395.

[18] D. Lakatos, F. Petit, and A. Albu-Schäffer, "Nonlinear oscillations for cyclic movements in human and robotic arms," IEEE Transactions on Robotics, vol. 30, no. 4, pp. 865-879, 2014.

[19] D. Lakatos, G. Garofalo, A. Dietrich, and A. Albu-Schäffer, "Jumping control for compliantly actuated multilegged robots," in IEEE Int. Conf. on Robotics and Automation, 2014, pp. 4562-4568.

[20] D. Lakatos and A. Albu-Schäffer, "Switching based limit cycle control for compliantly actuated second-order systems," in Proceedings of the 19th IFAC World Congress, 2014, pp. 6392-6399.

[21] G. A. Pratt and M. M. Williamson, "Series elastic actuators," in Intelligent Robots and Systems 95.'Human Robot Interaction and Cooperative Robots', Proceedings. 1995 IEEE/RSJ International Conference on, vol. 1. IEEE, 1995, pp. 399-406.

[22] J. Pratt and B. Krupp, "Design of a bipedal walking robot," in SPIE Defense and Security Symposium. International Society for Optics and Photonics, 2008, pp. 69621F-69 621F.

[23] A. Seyfarth, S. Grimmer, D. F. B. Häufle, and K.-T. Kalveram, "Can robots help to understand human locomotion?" Automatisierungstechnik, pp. 653-661, 2012.

[24] M. H. Raibert, Legged Robots That Balance. The MIT Press, 1986.

[25] G. Garofalo, C. Ott, and A. Albu-Schäffer, "Walking control of fully actuated robots based on the bipedal slip model," in Proc. IEEE Int. Conf. on Robotics and Automation, 2012, pp. 1999-2004.

[26] M. W. Spong, "Modeling and control of elastic joint robots," Transactions of the ASME: Journal of Dynamic Systems, Measurement, and Control, vol. 109, pp. 310-319, 1987.

[27] P. Kokotovic, H. Khalil, and J. O'Reilly, Singular Perturbation Methods in Control: Analysis and Design. Academic Press, London, 1986.

[28] C. C. De Wit, G. Bastin, and B. Siciliano, Theory of robot control. Springer-Verlag New York, Inc., 1996.

[29] R. Featherstone, Rigid body dynamics algorithms. Springer Berlin, 2008, vol. 49

[30] K. Hunt and F. Crossley, "Coefficient of restitution interpreted as damping in vibroimpact," Journal of applied mechanics, vol. 42, no. 2 , pp. $440-445,1975$ 